\title{
О филогенетическом положении некоторых подсемейств в семействе Ranunculaceae Juss.
}

\section{On the phylogenetic position of some subfamilies in the family Ranunculaceae Juss.}

\author{
Евдокимов И. Ю. \\ Evdokimov I. Yu. \\ Алтайский государственный университет, г. Барнаул, Россия. E-mail: ivan.evdokimov.92@mail.ru \\ Altai State University, Barnaul, Russia
}

\begin{abstract}
Peфepam. Ботаническое семейство Ranunculaceae содержит важные лекарственные растения. Чтобы получить новые эволюционные свидетельства в отношении систематической классификации растений изучаемого семейства, мы использовали данные молекулярной биологии, а также данные исследований прошлых лет. В статье приводятся результаты филогенетического анализа трех родов семейства Ranunculaceae, систематическое положение которых всегда было противоречиво и дискуссионно: Coptis, Glaucidium, Hydrastis. Результаты анализа не только поддержали монофилию каждого из этих родов, но также указывали на ряд дополнительных моментов, что, в свою очередь, подтверждает данные наших исследований.
\end{abstract}

Ключевые слова. Молекулярный анализ, филогения, Coptis, Glaucidium, Hydrastis, Ranunculaceae.

Summary. The botanical family Ranunculaceae includes important medicinal plants. To obtain new evolutionary evidence regarding the systematic classification of plants of the studied family, we used molecular biology data, as well as data from past studies. This article presents the results of phylogenetic analysis of three genera of Ranunculaceae, the systematic position of which has always been controversial and debatable: Coptis, Glaucidium, Hydrastis. The results of the analysis not only supported the monophyly of each of these genera, but also indicated a number of additional points, which in turn confirms the data of our studies.

Keywords. Coptis, Glaucidium, Hydrastis, molecular analysis, phylogeny, Ranunculaceae.

Ranunculaceae - семейство цветковых растений, которое включает в свой состав 61 род и около 2500 видов (Евдокимов, 2015). Хотя представители изучаемого семейства распространены во всем мире, растения наиболее часто встречаются в умеренных и холодных районах Северного полушария. Изучаемое семейство содержит много декоративных растений, большое количество представителей фармацевтически важны, некоторые из них имеют подтвержденную лекарственную ценность. Растения семейства Ranunculaceae имеют сложные химические составы, многие из которых представляют важные таксономические характеристики, и одни и те же химические компоненты распределяются между разными родами (Peng et al., 2006). Это, в свою очередь, также является филогенетически важным признаком (Ro et al., 1997). Исследователи использовали различные таксономические характеристики для определения важности связей между филогенезом, химическим составом и даже фармакологическими свойствами. Однако филогенетические связи между родами в семействе Ranunculaceae, особенно принадлежность к нескольким родам, противоречивы. Многие ученые выполняли ряд исследований по данной теме, применяя разные методы. Результаты их исследований частично совпадают с нынешней классификацией. В данной работе хотим показать самостоятельность родов Coptis Salisb., Glaucidium Siebold et Zucc, Hydrastis J. Ellis относительно остальных родов семейства Ranunculaceae. Так как каждый из исследуемых родов образует самостоятельную ветвь на дендрограмме, мы предлагаем возвести каждую ветвь с одноименным названием рода в ранг подсемейства. 
При проведении молекулярно-филогенетического анализа обнаружилось следующее: все описываемые в этой работе роды (Coptis, Glaucidium и Hydrastis) отходят от общего массива родов на дендрограмме и находятся близко друг к другу. В связи с этим возникла новая задача - исследовать морфологические характеристики данных родов, найти сходство и различие. На основе этих данных построена таблица.

Подсемейство Coptidoideae Tamura включает в настоящий момент три рода, систематическое положение которых дискуссионнно. Общие морфологические признаки цветков (двойной околоцветник из лепестковидных чашелистиков и более мелких трубчатых лепестков, плодолистики с несколькими семязачатками) и плодов (листовки) их видов присущи также другим родам семейства Ranunculaceae, именно поэтому данные роды были предметом дискуссий и меняли свое таксономическое положение время от времени. Так, род Xanthorhiza L. входил в состав семейства Paeoniaceae, трибы Helleboreae (Prantl, 1887) и Isopyreae (Lotsy, 1911); род Coptis Salisb. рассматривался в составе триб Helleboreae (Bentham, Hooker, 1862; Prantl, 1887) и Isopyreae. Ранее в состав данного подсемейства входил еще и род Asteropyrum Drumm. et Hutch, сейчас же он входит в состав подсемейства Calthoideae. Анатомические (Lotsy, 1911), кариологические (Langlet, 1932; Gregory, 1941) и палинологические данные подтвердили близость родов Xanthorhiza и Coptis, так как сосудистые пучки у их видов расположены по кругу, не образуя цилиндр; их виды принадлежат к одному кариотипу (мелкие хромосомы, основное число $x=9$ или 18). Кроме того, виды обоих родов содержат бербередин, отсутствующий у представителей других родов Ranunculaceae. Жизненные формы их различны, но есть общие признаки морфоструктуры побегов.

Следующее исследуемое подсемейство Glaucidioideae (Tamura) Loconte. Единственный представитель подсемейства Glaucidioideae - Glaucidium palmatum, монотипический род и вид, распределенные на Хоккайдо, Северном Хонсю и стороне Японского моря в Центральном Хонсю, был описан Р. F. V. Siebold и J. G. Zuccarini (1845). Они отнесли его в Ranunculaceae. Янчэн (Janchen, 1948) поднял ранг подсемейства Hydrastidoideae и включил в него Glaucidium. Было несколько авторов, которые рассматривали Glaucidium и Hydrastis в качестве членов Berberidaceae. Лоци (Lotsy, 1911) и Ланглет (Langlet, 1932) классифицировали оба рода в Podophylloideae сем. Berberidaceae. Химмельбаур (Himmelbaur, 1913) отделил Glaucidium и Hydrastis от Podophylloideae и предложил подсемейство Glaucidioideae перенести в Berberidaceae, что также сделал Miуaji (1930), хотя он использовал другое название подсемейства - Hydrastidoideae.

Glaucidium, Hydrastis имеют много общих признаков: 1) хорошо развитый ползучий корень; 2) один радикальный лист на конце корневища; 3) цветоносные стебли, не имеющие радикальных листьев и т. д.

При использовании методов молекулярной биологии просматривается довольно ясная картина: p. Glaucidium входит в состав семейства Ranunculaceae, в меньшей степени он имеет родство с семействами Pаeoniaceae и Berberidaceae. Но и в семействе Ranunculaceae p. Glaucidium образует отдельную ветку на дендрограмме, то есть род является довольно архаичным, и его отделение от остального набора родов изучаемого семейства произошло гораздо раньше. Поэтому он по праву занимает свое ранговое место подсемейства.

Далее речь пойдет еще об одном подсемействе - Hydrastidoideae Rafn., имеющем монотипный род Hydrastis, который встречается только в умеренных широтах Северной Америки. Он был одним из самых противоречивых родов в порядке Ranunculales с точки зрения его систематического положения. Род был впервые признан Линнеем (Linnaeus, 1759); после того, как Джусье (Jussieu, 1789) посчитал его членом семейства Ranunculaceae, Hydrastis оставался в этом семействе до конца XIX в. К тому времени стало ясно, что Hydrastis имел довольно много сходства с Podophyllum L. и Diphylleia Michx. (Tischler, 1902), род был включен в состав семейства Berberidaceae Engler (Мияджи, 1930). Позже Hydrastis был снова перемещен в Ranunculaceae, так как род имеет большое сходство с родом Glaucidium, а не с Podophyllum и Diphylleia семейства Berberidaceae (Janchen, 1948). Glaucidium почти всегда считался членом Ranunculaceae. В настоящее время большинство общих методов изучения включают как Hydrastis, так и Glaucidium в семейство Ranunculaceae в качестве подсемейств Glaucidioideae или Hydrastidoideae (Buchheim, 1964; Cronquist, 1981). Лемесле (Lemesle, 1950) впервые предложил 
㞼

\begin{tabular}{|c|c|c|c|c|}
\hline & 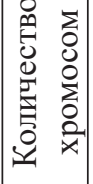 & $\underset{\|}{\ddot{x}}$ & $\underset{\varkappa}{\stackrel{ }{\|}}$ & $\underset{\|}{\|}$ \\
\hline & 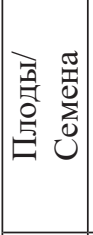 & 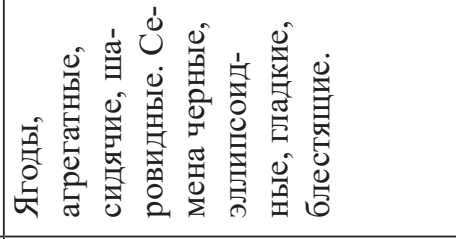 & 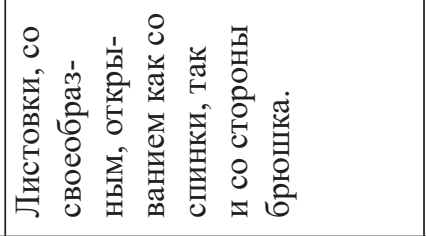 & 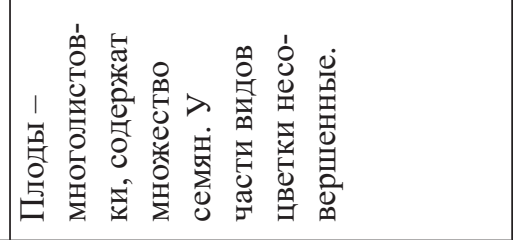 \\
\hline & 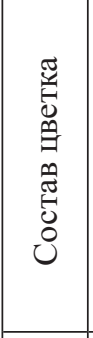 & 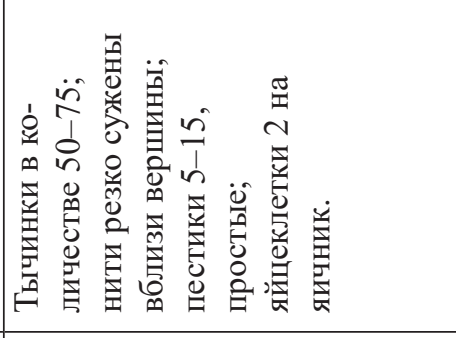 & 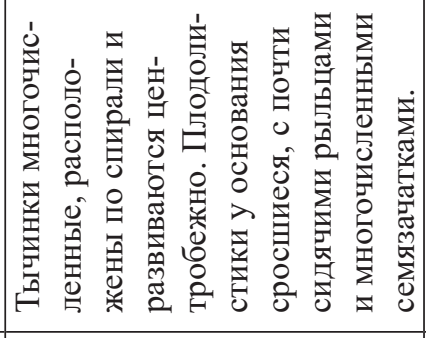 & 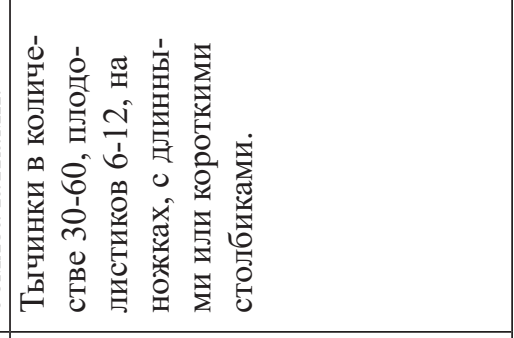 \\
\hline 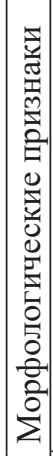 & 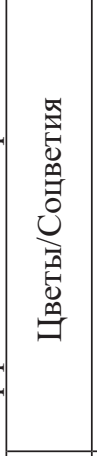 & 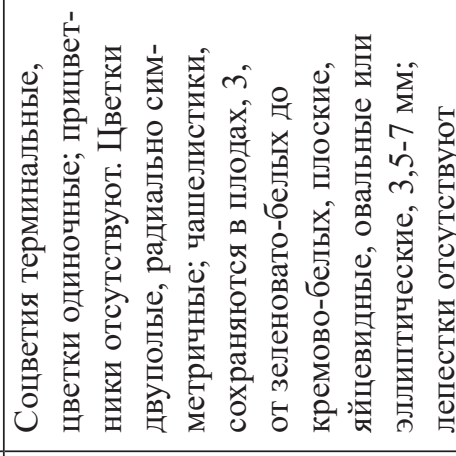 & 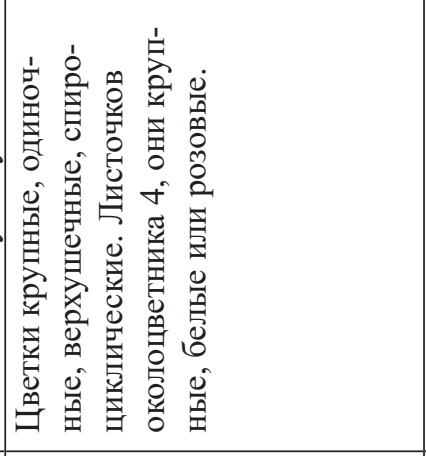 & 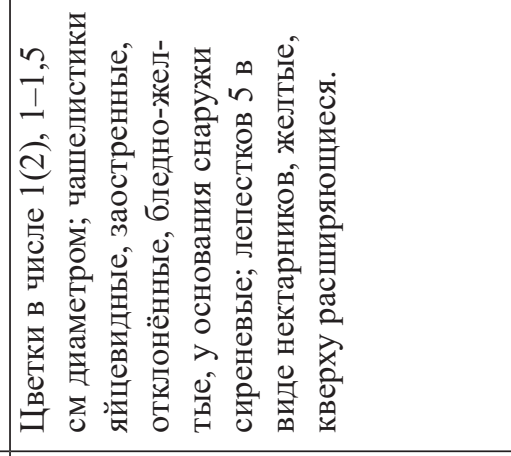 \\
\hline & 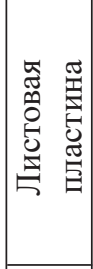 & 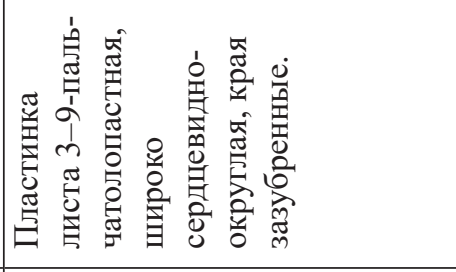 & 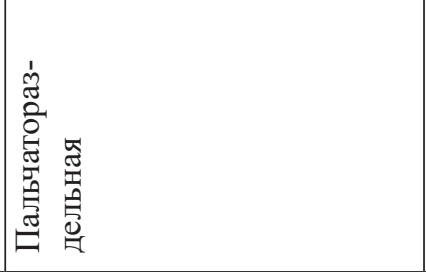 & 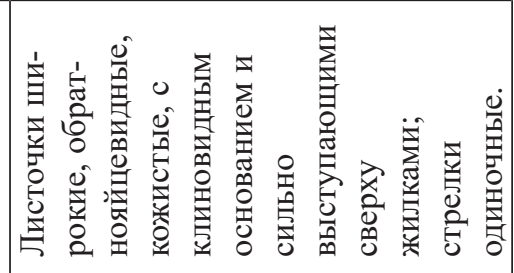 \\
\hline & 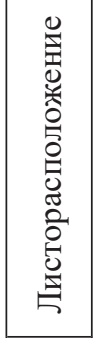 & 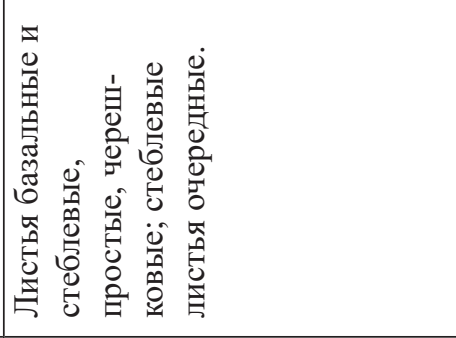 & 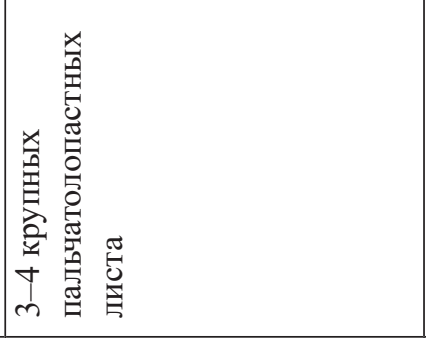 & 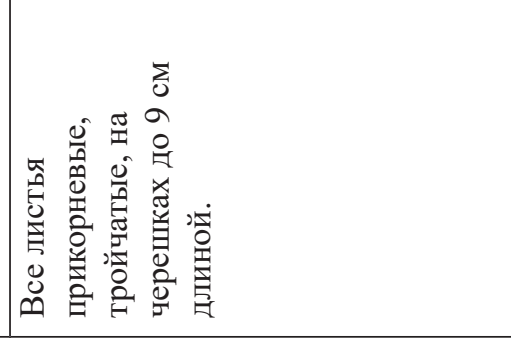 \\
\hline & 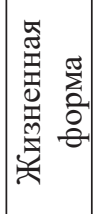 & 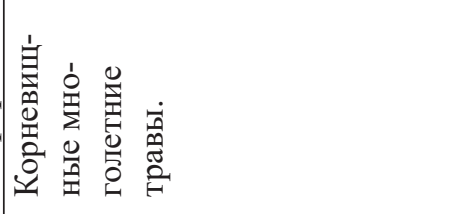 & 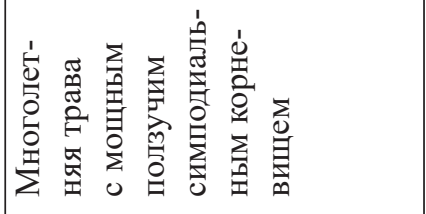 & 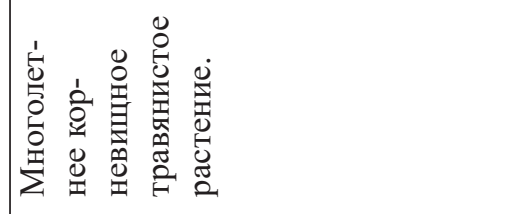 \\
\hline & $\stackrel{7}{\circ}$ & 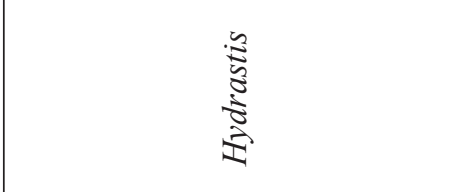 & 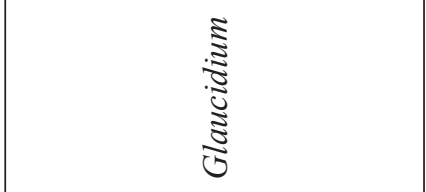 & $\frac{\tilde{\Xi}}{\delta^{2}}$ \\
\hline
\end{tabular}


установить это унигенерическое семейство Hydrastidaceae, по названию рода, потому что у Hydrastis есть скалярные перфорации сосудов, вместо исключительно простых, общих для Ranunculaceae, хотя он не исследовал перфорации сосудов в Glaucidium. Это предложение когда-то было принято А. Л. Тахтаджяном (1966), но, в дополнение к простым, сетчатые перфорации сосудов были позже найдены и у представителей рода Glaucidium (Tamura, 1972).

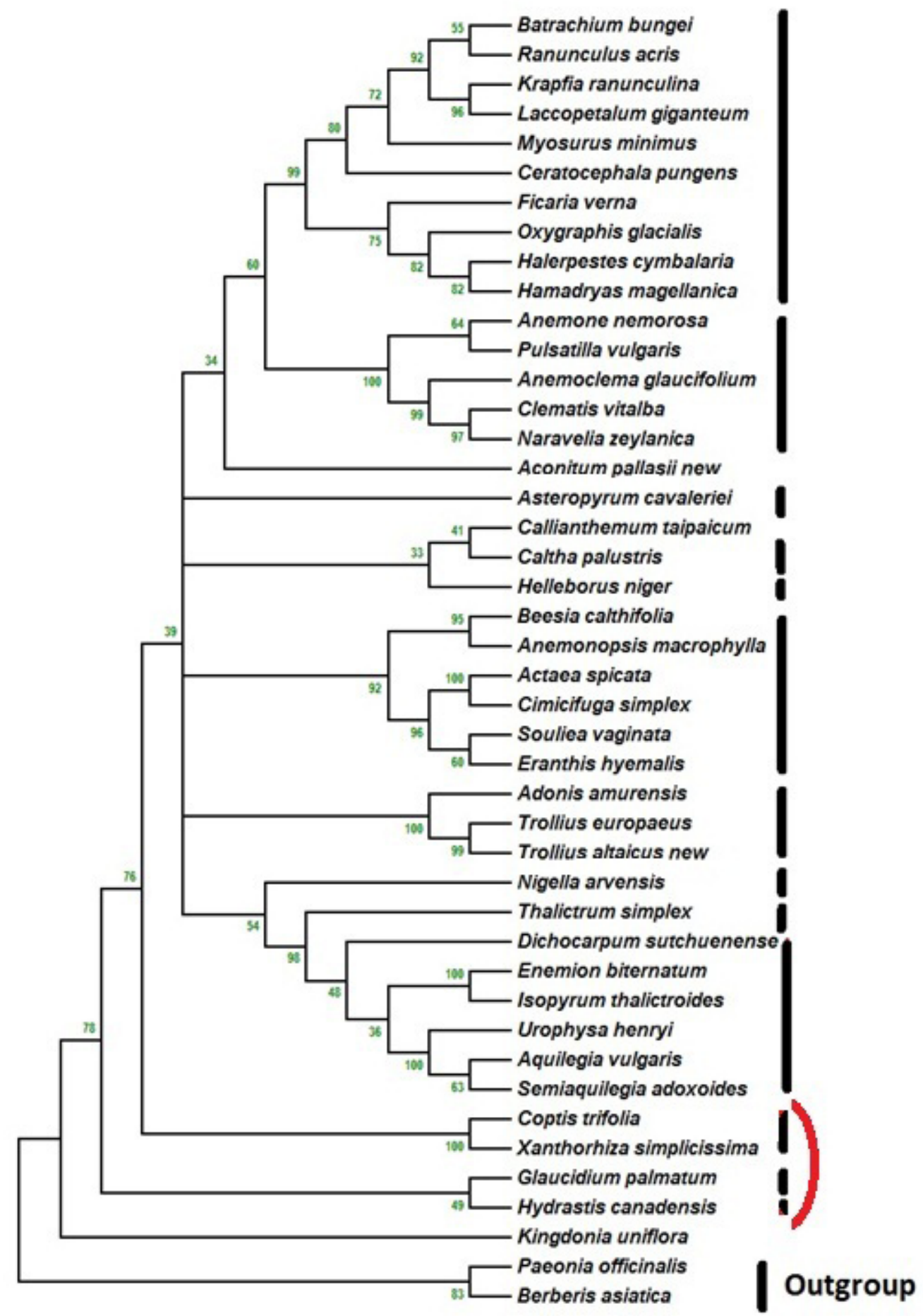

Рис. 1. Дендрограмма, построенная методом ближайших соседей (Neghbour-Joining) с помощью программы MrBayes 3.0.4 для trnl-f фрагментов флоропластной ДНК между 60 представителями семейства Ranunculaceae и 2 представителей родственных семейств, в качестве Outgroup. Филогенетическое родство менее 50 \% между представителями не отображено на дендрограмме. Сиквенсы, используемые для построения дендрограммы, взяты в генбанке NCBI, а также добавлены последовательности, секвенированные нами. 


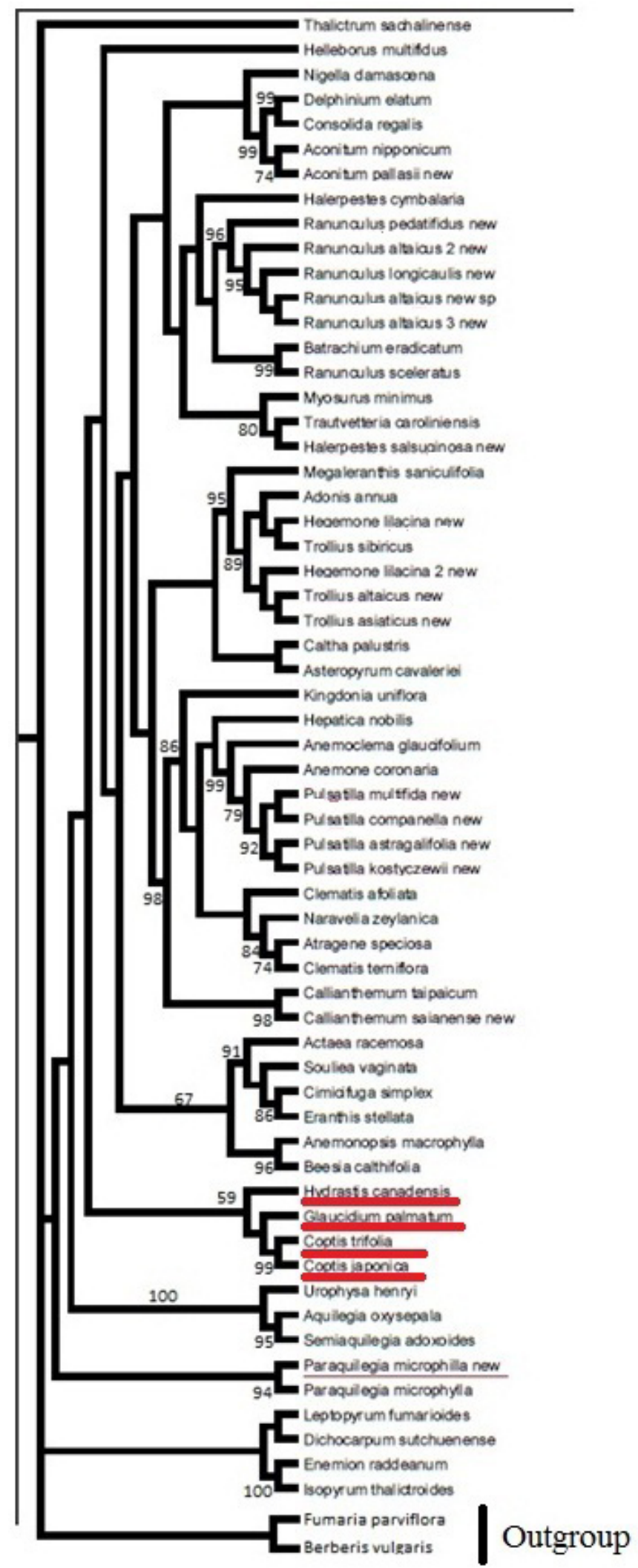

Рис. 2. Дендрограмма, построенная методом ближайших соседей (Neghbour-Joining) с помощью программы MEGA 7 для rbcL фрагментов флоропластной ДНК между 42 представителями семейства Ranunculaceae и 2 представителя родственных семейств (Outgroup). Филогенетическое родство менее 30 \% между представителями не отображено на дендрограмме. Сиквенсы, используемые для построения дендрограммы, взяты в генбанке NCBI, а также добавлены последовательности, секвенированные нами. 
Общее сравнение, основанное на морфологических, анатомических, цитологических, хемотаксономических и палинологических признаках, а также данных молекулярной биологии не подтвердило включение Hydrastis в другие семейства, кроме как в Ranunculaceae. В заключение, учитывая его особенности, такие как более высокое гаплоидное число хромосом $(n=13)$, морфологию пыльцы, а также его менее специализированные характеристики, можно сделать вывод, что ветвь, включающая в себя Hydrastis, представляет собой реликтовую примитивную группу, которая очень рано разошлась с общим родовым деревом сем. Ranunculaceae.

\section{Заключение}

Ранние исследования показывают, что основная часть родов была распределена в разные группы на основе сравнительно морфологического анализа. Для исследования филогении семейства нами строились дендрограммы на основе данных молекулярной биологии. Наши деревья, построенные на основе последовательностей ядерных участков ДНК, наглядно разделяются на группы (рис. 1, 2). Генетическая поддержка разделения на клады различна, но вполне достаточна для определения их в разные филогенетические группы.

На основе современных знаний о морфологических, кариологических, химических и молекулярных свойствах, мы показали, что систематика и классификация родов Coptis, Glaucidium и Hydrastis, включенных в Ranunculaceae, должны быть пересмотрены. Каждый представленный в статье род по праву должен быть родоначальником самостоятельной реликтовой группы, которая, в свою очередь может рассматриваться в качестве надродового таксона в ранге подсемейства.

\section{ЛИТЕРАТУРА}

Евдокимов И. Ю. Обзор систем семейства Ranunculaceae Juss. в хронологической последовательности // Проблемы ботаники Южной Сибири и Монголии: сб. науч. статей по материалам XIV междунар. науч.-практ. конф. Барнаул, 2015. - С. 140-144.

Taxтаджян А. Л. Система и филогения цветковых растений. - М.; Л.: Изд-во АН СССР, 1966. -610 с.

Bentham G., Hooker J. D. Ranunculaceae // Genera plantarum. - London, 1862. - Vol. 1. - P. $1-10$.

Buchheim G. Reihe Ranunculales // Syllabusder Pflanzenfamilien. Eds. H. Melchior, A. Engler's. 12 Aufl. 2. - Berlin: Gebrfider Borntraeger, 1964. - P. 131-147.

Cronquist A. An Integrated System of Classification of Flowering Plants. - New York: Columbia Univ. Press, 1981. - P. 111.

Gregory W. O. Phylogenetic and cytological studies in the Ranunculaceae Juss. // Trans. Amer. Phil. Soc., 1941. - Vol. 31, № 5. - P. 443-497.

Himmelbaur W. Die Berberidaceen und ihre Stellung im System. Denkschr. Akad. Wiss. Wien, Math.-naturw. K1., 1913. - № 89. - P. 733-796.

Janchen B. Die Systematische Gliederung der Ranunculaceen und Berberidaceen. - Klasse: Denkschr. Kaiserl. Akad. Wiss. Math.-Naturwiss., 1948. - P. 108.

Jussieu A. L. Genera plantarum secundum ordines naturales disposita, juxta methodum in Horto Regio Parisien siexaratum, anno M.DCC.LXXIV., 1789. - P. 232.

Langlet C. Über Chromosomenverhältnisse und systematic der Ranunculaceae // Svensk. Bot. Tidakr., 1932. - Vol. 26, № 1-2. - S. 381-400.

Lemesle R. Hydrastis canadensis L. et sesprincipales falsifications // Rev. Gen. Bot., 1950. - № 57. P. 5-23.

Linnaeus C. Syst. Nat., 1759. - Vol. 2. - 1088 pp.

Lotsy J. P. Ranunculaceae // Vortrage iiber Botanische Stammesgeschichte. - Jena: Verlag von Fischer, 1911. - Bd. 3. - S. 567-587.

Miyaji Y. Beitrage zur Chromosomenphylogenie der Berberidaceen // Planta, 1930. - № 11. - P. 650-659.

Peng Y., Chen S. B., Chen S. L., Xiao P. G. Preliminary pharmaphylogenetic study on Ranunculaceae China // J. Chin. Materia Medica, 2006. - Vol. 31. - P. 1124-1128.

Prantl K. Beitráge zur Morphologie und Systematik der Ranunculaceen // Bot. Jahrb., 1887. Vol. 9. - S. 225-273.

Ro K. E., Keener C. S., Mcpheron B. A. Molecular phylogenetic study of the Ranunculaceae: Utility of the nuclear 26S ribosomal DNA in inferring intrafamiliar relationships // Mol. Phyl. Evol., 1997. - Vol. 8. - P. 117-127. 
Siebold P. F. V., Zuccarini J. G. Florae Japonicae familiae naturales. Abh. Akad. - Muench., 1845. - № 4-2. - 184 p. Tamura M. Morphology and phyletic relationship of the Glaucidiaceae // Bot. Mag. Tokyo, 1972. - Vol. 85. - P. 2941.

Tischler G. Die Berberidaceen und Podophyllaceen. Versuche iner morphologisch biologischen, Monographie // Bot. Jahrb. Syst., 1902. - Vol. 31. - P. 596-727. 